\title{
COMMISSION 4: EPHEMERIDES
}

\author{
(EPHEMERIDES)
}

\author{
PRESIDENT: J. Chapront \\ VICE-PRESIDENT: G.A. Krasinsky \\ ORGANIZING COMMITTEE: V.K. Abalakin, J.-E. Arlot, T. Fukushima, \\ M.-F. He, C.Y. Hohenkerk, G.H. Kaplan, H. Kinoshita, J.H. Lieske, \\ H. Schwan, P.K. Seidelmann \& M. Standish
}

\section{IAU COMMISSION 4 GENERAL REPORT}

The commission has a website at Paris Observatory (http://syrte.obspm.fr/iaucom4/). The previous website at JPL (http://ssd.jpl.nasa.gov/iau-comm4/) is still operating and updated.

\section{INSTITUTIONAL REPORTS}

\subsection{Royal Greenwich Observatory / HMNAO}

HMNAO is now based at the Rutherford Appleton Laboratory under the auspices of the Council for the Central Laboratory of the Research Councils. It continues to operate on a commercial basis funded by royalty and contract income with three staff reporting to PT Wallace.

Joint publications with the US Naval Observatory, The Nautical Almanac, The Astronomical Almanac and Astronomical Phenomena, have been produced on schedule. The Astronomical Almanac Online has been produced as a companion to the printed book and is available at http://asa.usno.navy.mil and http://asa.nao.rl.ac.uk in conjunction with the US Naval Observatory.

New editions of Planetary and Lunar Coordinates 2001-2020 and Navpac and Compact Data 2001-2005 have been produced. U.S. editions of these publications have been produced by Willmann-Bell, the latter being known as AstroNavPC and Compact Data 2001-2005. New versions of Sight Reduction Tables for Air Navigation have been produced under the title Rapid Sight Reduction Tables for Navigation to reflect their use by mariners. Lunar distance data were produced for the first time since the beginning of the last century in almanac form for the BBC program "The Ship" in a recreation of Cook's first voyage to the Pacific.

Expansion of web information services has been funded and will be implemented over the next six months. This will replace the Websurf service currently available to the National Marime Museum and will be available to the general public.

\subsection{U.S. Naval Observatory}

A. Fiala retired as Chief of the Nautical Almanac Office (NAO) in June 2000. S. Howard replaced him in October 2000. G. Kaplan served as Acting Chief in the interim.

Publication of The Astronomical Almanac (AsA), The Nautical Almanac, The (U.S.) Air Almanac, and Astronomical Phenomena continued as a joint activity between Her Majesty's Nautical Almanac Office of the United Kingdom and the U.S. Nautical Almanac 
Office. The Astronomical Almanac Online, a Web-based complement to the printed book, began operation in January 2002.

Work on MICA 2.0, a major upgrade of the Multiyear Interactive Computer Almanac, is in progress.

Preliminary ephemerides for 78 asteroids were produced for use in the AsA. The current plan is to improve these preliminary ephemerides and release them as a new product.

Considerable time was spent understanding the IAU 2000 resolutions and how they will be implemented in the almanacs and other products. Work on a new theory of precession, to complement the IAU 2000 nutation model, is underway.

Use of the Astronomical Applications Department web site (http://aa.usno.navy.mil/) grew significantly during the reporting period.

\subsection{Astronomisches Rechen-Institut}

The data of the annually published "Apparent Places of Fundamental Stars" were provided for the years 2000,2001 and 2002 via the internet and additionally in a small booklet where examples for a few stars are merely given. The printed extensive volumes prior to the year 2000 could no longer be published for economical and scientific reasons. The following main changes were introduced starting with the data for the year 2000:

1) Replacement of the former extensive volumes by a small booklet, giving the introductory explanations and apparent places for a few stars as examples.

2) The data for many more stars are given via the internet (http://www.ari.uniheidelberg.de/ariapfs/ ).

3) The apparent places are tabulated day by day for all stars. They are given with and without the short period terms or nutation.

4) The basic data for the apparent places are taken from the FK6 catalogue and from the Hipparcos catalogue. Both versions are offered via the internet.

\subsection{Institute of Applied Astronomy}

The program system ERA for ephemeris and dynamical astronomy based on a dedicated problem-oriented programming language has been updated. With this system as a tool Krasinsky G.A., Pitjeva E.V., Vasilyiev M.V. have generated new numerical ephemerides EPM2002 of the major planets and the Moon. In the dynamical model the perturbations from 300 largest asteroids are taken into account by simultaneous numerical integration of equations of motion of the major planets, Moon and the asteroids. Parameters of the ephemerides are fitted to the observations used in DE405/LE405 including a large volume of more recent data from the server of JPL supported by M. Standish.

While processing observations of satellites of the major planets, their dynamical theories have been constructed by numerical integration, parameters of the satellite systems being improved in the general analysis of the observational data. Lunar part of the ephemerides is based on Lunar Laser Ranging data for the time interval 1969-2001. Some refinements of the model of the Moon's rotation are made to account for effects of the lunar fluid core. The ephemerides are presented in the form of Chebyshev polynomials for both the TDB and TCB time scales. DOS version ERA-7 with documentation and the LLR applications is available via anonymous FTP quasar.ipa.nw.ru/incoming/era

Sveshnikov M.L. has rediscussed observations of transits of Mercury through the solar disk and detected oscillations of the apparent solar radius with the period of solar activity 11 years (the maximums are at the epoch of maximums of solar activity).

Lukashova M.V., and Rumjantzeva L.I. have prepared and published Canon of solar eclipses in Russia for the years 1000-2050.

In Department of Astronomical Yearbook (in charge of Sveshnikov M.L.) a revised version of Russian Astronomical Yearbook is under preparation to be based on the Hippar- 
cos/FK6 systems for stars and DE405/LE405 for planetary ephemerides. New versions of Nautical Almanac MAA-2 for 2002-2003 have been published.

\subsection{National Astronomical Observatory of Japan}

The Ephemeris Computation Office of the Public Relations Center, NAOJ, has completely updated the Japanese civil almanac named Ephemeris Yearbook from the year 2003. The major points of revision are as follows:

1) The position and velocity of the Sun, the Moon, and major planets are replaced from DE200/LE200 to DE405/LE405 (Standish 1998). As a by-product, the fundamental reference frame of the Yearbook has shifted from the mean equinox and equator at J2000.0 to ITRF. Also the system of astronomical constants has changed to the IAU 2000 Best Estimates of Astronomical Constants (Fukushima).

2) The IAU 1980 nutation theory was replaced to SF2001 (Shirai and Fukushima), whose difference from IAU2000A is small as less than 1.5 mas in longitude and 0.6 mas in obliquity for the forthcoming 20 years.

3) To be conformal with the modification of the Japanese laws of the surveying and the hydrographic duties enforced on April 1st, 2002, we revised the data of geodetic system and the longitude and latitude of many landmarks in Japan to those in the ITRF.

4) In order to obtain more precise values of the nutations and the equation of equinox, we changed their tabulation step size from 10 days to 4 days.

\subsection{Paris Observatory Lunar Analysis Center}

The group POLAC (Paris Observatory Lunar Analysis Center - SYRTE) performs reduction and analysis of LLR observations, in connection with the laser station at Grasse (CERGA - Observatoire de la Cote d'Azur).

In 2001, a set of lunar and solar orbital parameters and free libration parameters have been determined with a fit to LLR observations covering the time span [1972-2001] and using all the observing stations (McDonald, Haleakala and CERGA). Besides, a determination of the orientation of the ecliptic inertial reference frame with respect to the IERS reference system, and to the dynamical equatorial reference frames has been obtained. These results gathered in a solution S2001 have been introduced in the analytical ELP series providing new ephemerides of the orbital motion of the Moon, and libration.

In parallel a new construction of the planetary perturbations in ELP has been realized which has been compared on the long range to DE406 and leading to the new analytical version of ELP named ELP/MPP02.

\subsection{The Astronomical Institute of the Czech Republic}

The Astronomical Institute, Academy of Sciences of the Czech Republic, in close cooperation with the Observatory and Planetarium of Prague, issues each year an astronomical yearbook of about 250 pages for amateur astronomers (in Czech) "Hvězdářská ročenka", with a limited precision. The yearbook is based on the VSOP82 ephemerides and it contains the ephemerides of the Sun, Moon, planets and their satellites, asteroids, comets, meteoric streams and variable stars. Many phenomena (such as eclipses, occultations, conjunctions and oppositions etc...) are also included. The book is accompanied by a diskette with some ephemerides of higher precision. All calculations are done at the Astronomical Institute and, in addition, the prediction of occultations is provided also for four Romanian observatories and published in their astronomical yearbook. 


\subsection{Jet Propulsion Laboratory / Caltech}

The Solar System Dynamics group continues under the supervision of D K Yeomans as part of JPL's Navigation Section. The group continues to support JPL's spacecraft navigation with the constant updating of the ephemerides.

The group's website, "http://ssd.jpl.nasa.gov/", provides various astronomical tools and constants; it also includes "Horizons", an interactive provider of customized ephemerides and related information for all of the major planets, natural satellites, numbered asteroids, and comets, using JPL's latest high-accuracy ephemerides.

DE405 and its extended version, DE406, remain the latest export planetary and lunar ephemerides, since they are available on CD and form the basis of the Astronomical Almanac. The complete set of observations, fit by the planetary ephemerides, are available at "http://ssd.jpl.nasa.gov/plan-eph-data/index.html".

Element-type formulae exist for all of the minor satellites, while higher precision ephemerides have been integrated for the major ones, extending over the past few decades and extending into the future for a decade or two.

Ephemerides for comets and asteroids are created, as needed, using integrations fit to all available observations.

Orbital computations for any newly observed objects - NEO's, etc. - are made on an ad hoc basis.

\subsection{Institut de Mécanique Céleste et de Calcul des Ephémérides - Bureau des Longitudes}

1) Printed ephemerides:

Connaissance des temps is published yearly as previously together with supplements dedicated to Natural Planetary Satellites. An explanatory supplement "Introduction aux ephemerides astronomiques" contains all the theoretical information on the making of ephemerides. Ephemerides of asteroids and comets are published continuously in the Notes Scientifiques et Techniques. The Annuaire du Bureau des longitudes is published yearly as previously and is dedicated for general public use. The Ephemerides Nautiques are published each year for navigation.

2) Ephemerides under electronic form:

Softwares providing the ephemerides of Sun, Moon, planets, satellites, asteroids and comets are available on the FTP server of IMCCE at ftp.imcce.fr together with the prediction of phenomena. IMCCE provides also on-line ephemerides for a large public through Internet for most of the objects of the solar system providing geocentric as well as topocentric positions in several reference frames for different theoretical models and ephemerides for physical observations together with a graphic representation of the surfaces of the bodies. Predictions of the eclipses of the Sun and the Moon are also available.

3) International ephemerides distribution:

Predictions of the Galilean satellites phenomena and their configurations are still sent yearly to several ephemerides offices and astronomical laboratories. These data are also available through FTP.

4) Modifications arising in a next future:

The theoretical models used in the ephemerides will be changed in a next future in order to increase the accuracy of the published data. The recommendations of IAU will be introduced, first, only in the ephemerides under an electronic form, in order to help the users to understand the modifications. Note that the address of the web site of the Institut de mecanique celeste is now: http://www.imcce.fr

J. Chapront

President of the Commission 